\title{
BRCA1 et BRCA2 : des échafaudages de réparation des lésions de l'ADN ?
}

Les mutations germinales des gènes BRCA1 et BRCA2 rendent comptent d'environ la moitié des syndromes familiaux de cancers du sein et de l'ovaire. Ces deux gènes feraient partie d'une famille fonctionnelle de gènes appelés caretakers, capables d'informer la cellule sur l'existence de lésions dans son $\mathrm{ADN}$ et, éventuellement, de participer aux processus de réparation [1]. Cette hypothèse repose en particulier sur l'observation d'une association des protéines BRCA1 et BRCA2, dans les cellules somatiques et dans les cellules méiotiques avec de multiples acteurs des processus de réparation de l'ADN, en particulier RAD51, homologue eucaryote de RecA d'E. coli qui participe à l'échange de brins lors de la recombinaison homologue. Cependant, les fonctions biochimiques précises des protéines BRCA1 et BRCA2 dans les processus de réparation restent inconnues. Plusieurs publications récentes identifient ces protéines comme des composants majeurs d'échafaudages de réparation des lésions de l'ADN.

L'interaction de BRCA1 avec de multiples partenaires protéiques impliqués dans les mécanismes de réparation de l'ADN avait été illustrée par sa présence dans un complexe nucléaire multiprotéique de plusieurs mégadaltons appelé BASC (pour BRCA1-associated genome surveillance complex) [2]. De même, Marmorstein et al. ont récemment décrit la présence de BRCA2 dans un complexe multiprotéique de même nature [3]. Les auteurs identifient en outre, dans ce complexe, une protéine (BRAF35 pour BRCA2-associated factor 35) capable de se fixer à des structures d'ADN cruciformes de la réplication et de la réparation. Le complexe BRCA2-BRAF35, qui est associé à la chromatine condensée au cours de la mitose, pourrait ainsi participer au processus de ségrégation des chromosomes.

$\mathrm{Si}$ on sait maintenant que les protéines BRCA sont susceptibles d'interagir avec de nombreux partenaires protéiques, les données fonctionnelles sont encore rares. Elles suggèrent toutefois que ces protéines puissent jouer un rôle dans l'organisation des corps nucléaires.

Ainsi, Garcia-Higuera et al. ont démontré l'existence d'une interaction fonctionnelle entre BRCA1 et le produit du gène $F A N C D 2$, l'un des six gènes dont les mutations sont responsables de l'anémie de Fanconi [4]. L'exposition à des agents génotoxiques provoque la migration de la protéine FANCD2, dont la répartition nucléaire est normalement diffuse, vers des corps nucléaires. Deux événements au moins déterminent cette migration: d'une part, la présence de la protéine BRCA1 qui, dans ces circonstances, se co-localise avec FANCD2 dans les corps nucléaires et, d'autre part, la monoubiquitinylation de la protéine FANCD2. Or, on sait que BRCA1 a une activité E3 ubiquitine ligase in vitro qui implique son domaine doigt de RING, et l'on peut donc envisager que ce soit BRCA1 qui contrôle la mono-ubiquitinylation de FANCD2. Cette interaction entre les protéines FANCD2 et BRCA1, provoquée par des lésions génotoxiques, n'est cependant démontrée par co-immunoprécipitation qu'avec des anticorps anti-BRCA1, et malheureusement pas dans l'autre sens. Toutefois, ces résultats suggèrent fortement que BRCA1 puisse jouer un rôle important dans l'organisation des corps nucléaires et y recruter FANCD2.

Un autre exemple vient d'être fourni par le groupe de Livingston qui a identifié un nouveau partenaire de BRCA1 capable de se fixer à son extrémité carboxy-terminale sur les motifs répétés BRCT (BRCA1 C-terminal) [5]. Il s'agit d'un membre de la famille des hélicases à ADN contenant un domaine DEAH, et dont l'un des représentant est le gène $X P D$ dont les mutations sont responsables du Xeroderma pigmentosum (groupe D) (m/s 1998, $\left.n^{\circ} 11, p .1289\right)$. Cette protéine, BACH1, semble jouer un rôle dans la réparation des cassures double brin comme co-facteur de BRCA1. Tout comme FANCD2, BACH1 n'est localisée dans des corps nucléaires que si BRCA1 est présente. En outre, BACH1 pourrait représenter un nouveau gène de susceptibilité au cancer du sein, car une mutation germinale de BACH1 située dans son domaine hélicase a été mise en évidence dans deux cas de cancer du sein d'apparition précoce.

Qu'en est-il de BRCA2 ? Davies et al., en étudiant les conséquences fonctionnelles de l'interaction entre BRCA2 et RAD51, observent qu'un peptide représentant l'une des huit unités répétées et très conservées de la protéine BRCA2 humaine (motifs BRC) est capable de séquestrer RAD51 et d'empêcher son interaction avec l'ADN [6]. En cas de lésions de l'ADN, le complexe BRCA2-RAD51 est dirigé vers ces lésions, et la fonction de RAD51 dans la réparation est activée. Les formes mutantes de BRCA2, qui ont perdu leur signal de localisation nucléaire mais conservé les motifs BRC, semblent capables de séquestrer RAD51 
dans le cytoplasme, l'empêchant ainsi de répondre aux alarmes signalées par les lésions de l'ADN. Les auteurs n'apportent cependant pas la preuve formelle d'une interaction directe entre BRCA2 et RAD51 dans le compartiment cytoplasmique. Ces résultats suggèrent toutefois que BRCA2 puisse être un convoyeur de l'appareil de réparation, dont RAD51, vers les lésions de l'ADN. En accord avec cette hypothèse, les cellules qui expriment des formes mutantes de BRCA2 sont radiosensibles et présentent une instabilité génétique exacerbée se manifestant par une fréquence élevée de réarrangements chromosomiques spontanés. Argument supplémentaire, les cellules humaines $B R C A 2^{\text {mut } / \text { mut }}$ ont un défaut net de réparation des cassures double-brin par conversion génique [7]. Certes la démonstration souffre ici de l'absence de complémentation fonctionnelle par réintroduction de l'allèle sauvage de BRCA2. Néanmoins, elle est confortée par des observations concordantes dans les cellules murines BRCA2 $2^{m u t / m u t}$. Cependant, chez la souris, il ne semble pas exister de séquestration cytoplasmique par BRCA2 car l'interaction de RAD51 avec le produit du gène muté BRCA2 est normale dans le noyau. Cette observation souligne les différences qui peuvent caractériser les processus impliquant BRCA1 ou BRCA2 entre l'homme et la souris.

L'ensemble de ces observations n'identifient toujours pas de fonction biochimique spécifique pour BRCA1 ou BRCA2. Néanmoins, elles convergent vers un concept «d'échafau- dage » de réparation que ces protéines contribueraient à assembler en réponse aux lésions génotoxiques. Ce concept avait déjà été évoqué pour BRCA1 par Scully et al. sur la base d'arguments génétiques montrant que la capacité de BRCA1 à complémenter le défaut de réparation des cellules $B R C A 1^{-/-}$était abolie par un ensemble hétérogène de mutations affectant divers domaines de la protéine, dont les effets étaient vraisemblablement plus structuraux que fonctionnels [8].

On peut souligner que l'implication de BRCA1 et BRCA2 dans des processus aussi différents que la réparation des cassures double-brin ou la réparation, couplée à la transcription, des lésions oxydatives, suggère que leur rôle n'est peut-être pas de participer stricto sensu à la réparation des lésions de l'ADN mais plutôt de reconnaître leur existence grâce aux altérations structurales qui leur sont associées. De même, alors que BRCA1 et BRCA2 sont présents dans les régions axiales des complexes synaptonémaux* (hors des synapses) au cours de la méiose mâle, il est peu probable qu'ils soient directement impliqués dans les événements de recombinaison méiotique qui se produisent dans les synapses. Il est plus vraisemblable qu'ils participent à la reconnaissance des cassures et/ou à la recherche d'homologies en préparation de la recombinaison méiotique.

\footnotetext{
* Complexe synaptonémal: structure des régions d'appariement des chromosomes au cours de la
} méiose.
1. Feunteun J. La prédisposition héréditaire au cancer du sein liée à BRCA1 et NRCA2: une maladie de la réponse aux lésions génotoxiques? Med Sci 1999; 15: 38-44.

2. Wang Y, Cortez D, Yazdi P, Neff N, Elledge SJ, Qin J. BASC, a super complex of BRCA1-associated proteins involved in the recognition and repair of aberrant DNA structures. Genes Dev 2000; 14: 927-39.

3. Marmorstein LY, Kinev AV, Chan GKT, et al. A human BRCA2 complex containing a structural DNA binding component influences cell cycle progression. Cell 2001; 104: 247-57.

4. Garcia-Higuera I, Taniguchi T, Ganesan S, et al. Interaction of the Fanconi anemia proteins and BRCA1 in a common pathway. Mol Cell 2001; 7 : 249-62.

5. Cantor SB, Bell DW, Ganesan S, et al. Bach1, a novel helicase-like protein, interacts directly with BRCA1 and contributes to its DNA repair function. Cell 2001; 105 : 149-60.

6. Davies AA, Masson JY, McIlwraith MJ, et al. Role of BRCA2 in control of the RAD51 recombination and DNA repair protein. Mol Cell 2001; 7 : 273-82.

7. Moynahan ME, Pierce AJ, Jasin M. BRCA2 is required for homology-directed repair of chromosomal breaks. Mol Cell 2001; 7: 263-72.

8. Scully R, Ganesan S, Vlasakova K, Chen J, Socolovsky M, Livingston DM. Genetic analysis of BRCA1 function in a defined tumor cell line. $\mathrm{Mol}$ Cell 1999; 4 : 1093-9.

\section{Jean Feunteun}

UMR 1599, Institut Gustave-Roussy, 39, rue Camille-Desmoulins, 94805 Villejuif Cedex, France.

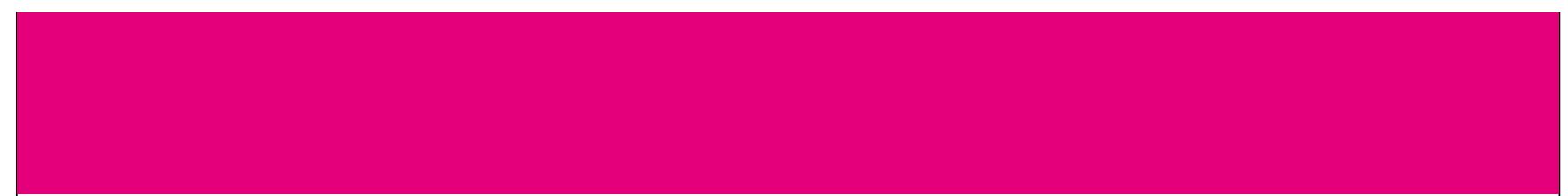

